\title{
Full Range Omnidirectional Sound Source for Near-Field Head-Related Transfer-Functions Measurement
}

\author{
BARTLOMIEJ CHOJNACKI, ${ }^{1,2}$ AES Student Member, SANG-IK TERRY CHO, ${ }^{1}$ AES Associate Member AND \\ (bchojnacki@agh.edu.pl) \\ (terry.cho@fb.com)
}

\author{
RAVISH MEHRA, ${ }^{1}$ AES Associate Member \\ (ravish.mehra@fb.com)
}

${ }^{1}$ Facebook Reality Labs, Redmond, WA, United States

${ }^{2}$ AGH University of Science and Technology, Cracow, Poland

\begin{abstract}
Head-related transfer-functions (HRTF) are a central part of spatializing audio. However measuring the near-field HRTF at close source distances presents unique challenges. In particular the existing sound sources designed to be appropriate for near-field HRTF measurements on human subjects exhibit a notable issue of being unable to generate a sufficient acoustic output level at lower frequencies (below $300 \mathrm{~Hz}$ ) while keeping a proper omnidirectional directivity pattern at higher frequencies. This paper proposes a novel design to overcome this limitation of low-frequency range. Several aspects of the design were considered in the paper: type of enclosure, low-frequency extension, choice of transducers, and metrics for sound source assessment. The chosen solutions are discussed together with numerical and experimental verification. The source constructed under the design method and process described herein achieved a frequency range of $120-16,000 \mathrm{~Hz}$ for which it can be used to measure HRTFs at source distances as small as $0.15 \mathrm{~m}$ from the subject's head.
\end{abstract}

\section{O INTRODUCTION}

Head-related transfer functions (HRTF) constitute an important part of many virtual auditory display systems for augmented and virtual reality applications, as they encode the binaural queues through which human listeners understand the spatial information of their auditory scene [1]. Although HRTF of human subjects can be obtained in a number of ways including numerical simulations, an accurate measurement is the only reliable way to establish the ground truth. The HRTF databases that are currently available and commonly used for binaural sound reproduction consist of far-field HRTF, which are defined for distances larger than $1 \mathrm{~m}$ [2]. Databases of near-field head-related transfer functions (NF HRTF), defined by the HRTF for source distances smaller than $1 \mathrm{~m}$, are comparably rare [3]. This can be attributed to the technical challenges of building an appropriate sound source for NF HRTF measurement, which have been investigated in several papers $[4,5]$.

For example, the previous work by Qu et al. [6] describes a method of measuring NF HRTF based on laser sparks, the suitability of which has not been demonstrated for human subjects. In discussing localization queues in NF HRTF, Brungart and Rabinowitz [7] describe a thin tube-based point source used in their measurement, for which they report the effective frequency range to be from $200 \mathrm{~Hz}$ to 15 $\mathrm{kHz}$. However this particular source shows a severe limitation in its output level at high frequencies, which introduces challenges in correctly measuring deep notches commonly found in HRTF at frequencies above several kilohertz. A recently published paper by Yu et al. [3] describes a database of NF HRTF on 56 human subjects. However they also report $300-350 \mathrm{~Hz}$ as the low-frequency response limit of the sound source used in their measurement system. The physical constraints of an acoustic measurement in the near-field of a human subject present a unique set of requirements for the sound source:

1. The sound source needs to provide a sufficiently high sound pressure level in a wide frequency range exceeding the noise floor of the data acquisition system and environment. Simultaneously it should be 
controllable to not exceed a dangerous output level for unprotected human ears.

2. The directivity of the sound source needs to be omnidirectional in order to accurately capture the reflection and diffraction around all parts of human anatomy, including head, ears, and torso.

3. The physical size of the source must be kept as small as possible in order to prevent the error introduced by multiple reflections between the head and source in the near-field, which is referred to as the multiscattering effect.

Based on the existing literature, no sound source has been reported to sufficiently and concurrently meet all of these requirements. Although a spherical sound source composed of many surface mounted transducers can be considered to be a practical solution that gets closest to meeting these requirements, the state-of-the-art [4] falls short in its lowfrequency response and omnidirectionality. In this paper a novel solution to overcome the challenges of a conventional spherical omnidirectional sound source is proposed in which the number and size of small, surface-mounted transducers is optimized for optimal omnidirectionality while a larger transducer subassembly is integrated inside the spherical structure with many small, distributed ports to extend the low-frequency range of the output.

The following sections of the paper describe the design process, leading to an optimized sound source with an extended frequency range. The example source constructed through this process achieved an optimized frequency and spatial response, which allows HRTF measurements over a wide frequency range of $120-16,000 \mathrm{~Hz}$ at source distances as short as $15 \mathrm{~cm}$ from the subject with a head radius of around $0.0875 \mathrm{~m}$. Some additional explanation of the maximum source size in relation to the subject's head size is provided.

\section{PROBLEMS WITH NEAR-FIELD HRTF MEASUREMENTS}

The current state-of-the-art solutions for near-field HRTF measurements are based on innovative technologies in omnidirectional acoustic sources. The limitations of such sources form the basis of this work. A study performed by Yu et al. led to the conclusion that the size of a spherical sound source at the distance of $0.15 \mathrm{~m}$ from the subject's head should be kept under a $35 \mathrm{~mm}$ radius in order to avoid spectral deviation error higher than $1 \mathrm{~dB}$ caused by the multi-scattering effect [8]. This conclusion was based on the study of two rigid spheres, one representing the sound source of different radius and the other one representing the head radius $8.75 \mathrm{~cm}$. However when we consider the head is not perfectly rigid and has some absorption, it would decrease the multi-scattering effect (the influence of reflections caused by both rigid source surface and surface of the measured subject). On the other hand, since the head radius may be even up to $0.135 \mathrm{~m}$ [9], the sound source should be taken into consideration with the actual subject's head size.
To get a wider scope on this topic Yu et al.'s work [8] was recreated in Facebook Reality Labs (FRL) with a finite element method (FEM) model of the same setup, considering spherical head and source to be perfectly rigid. Extending Yu's work, additional head sizes were considered up to a $0.135 \mathrm{~m}$ radius (the largest noted in anthropology). Spectral deviation error (SDE) was calculated, following an error rating method typical for $\operatorname{HRTF}[10,11]$ following Eq. (1):

$$
\operatorname{SDE}(f)=\left|20 \log _{10} \frac{|H(f)|}{|\hat{H}(f)|}\right|,
$$

where in this case $H(f)$ is the HRTF calculated with the sound source with a given size and $\hat{H}(f)$ is the HRTF measured with a point source (no size applied).

A detailed comparison of available source size vs. measurement distance for several head sizes $\left(\mathrm{H}_{\mathrm{s}}\right)$ varying from 0.075 to $0.135 \mathrm{~m}$ is shown in Fig. 1. The minimum measurement distance was defined as the distance above which the maximum SDE caused by multi-scattering in the whole frequency range was smaller than $1 \mathrm{~dB}$. The source size proposed by $\mathrm{Yu}$ et al. is indicated by a black dashed line to emphasize that the validity of the $0.2 \mathrm{~m}$ measurement distance holds for a portion of the possible range of human head sizes. Following these results, a $35 \mathrm{~mm}$ radius source should be suitable for measurements at the distance of $0.32 \mathrm{~m}$ for the largest head size, although measurements at roughly $0.15 \mathrm{~m}$ would be allowable for the average head size of $0.0875 \mathrm{~m}$.

A subsequent work by $\mathrm{Yu}$ et al. presented a physical prototype of an omnidirectional sound source [4]; the performance of this source was described as sufficiently accurate for near-field HRTF measurements. However the main issue of this design was its limited magnitude response, which was able to provide accurate SNR starting from 350 $\mathrm{Hz}$. In addition the measurement and analysis of its spatial response presented in the paper was made with several polar plots without broadband and strict assessment through full frequency range.

For some specific applications, there are commercially available sound sources (e.g., B\&K 4295, Microflown VVS) based on small tube openings that exhibit omnidirectionality in limited frequency bands. Many other forms of omnidirectional sound sources are known in the literature; for example an optimized horn approach [12], balloon sources [13], piezoelectric sources [14], and spark gap sources $[15,16]$. Some of the previously proposed designs are shown in Fig. 2.

These solutions, although innovative and practical for some applications, usually have many limitations when it comes to the measurement of near-field HRTF. For example using spark gap or laser sources causes a strong electromagnetic discharge that can be dangerous for microphones and human subjects. Horn-type and piezoelectric sound sources do not cover the full frequency range, so they need to be used as two or more separated devices at the same time, each covering a different frequency range. Balloon sources are difficult to operate and limited both in SPL and fre- 


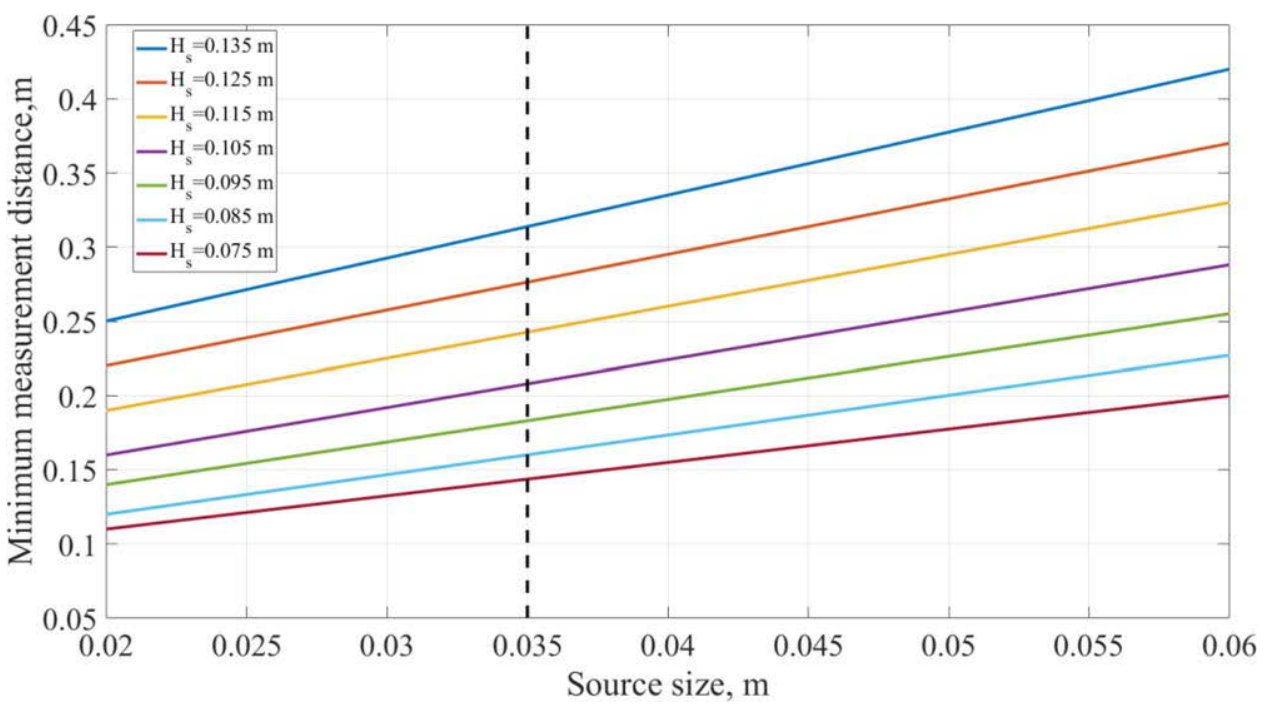

Fig. 1. Critical sound source size vs. measurement distances for head sizes from the range of $0.075-0.135 \mathrm{~m}$.

quency range. This in turn presents challenges for HRTF measurements of human subjects for which the total measurement time is critical for the comfort of the subject and ultimately the accuracy of the data.

Several papers in the literature describe sound sources consisting of multiple traditional electrodynamic loudspeakers, distributed evenly according to Platonic shapes. Leishman's work [18] describes a brief study on traditional Platonic shapes and their ratings with various directivity measures. There are also several analytical approaches to this topic [19-21]. However there is a lack of an in-depth study on the number of transducers and construction features of these sound sources. Therefore this paper focuses on the design of a spherical loudspeaker array with elements distributed according to a Platonic shape and addresses the details of its construction. The study on the sound source for NF HRTF measurements presented in this paper was aimed at the following:

- maximizing the omnidirectional performance,

- extending the frequency range achieved in the existing solutions,

- keeping the sound source as small as possible in order to minimize multi-scattering effect, and

- providing sufficient SPL output.

The main goal of this study was to optimize omnidirectional performance, construction, and acoustic aspects of a sound source of reduced dimensions. We focused on reducing the size of the enclosure and allowing the mounting of the biggest possible transducers in the enclosure; however some electroacoustic aspects of speakers working in small a)



e)

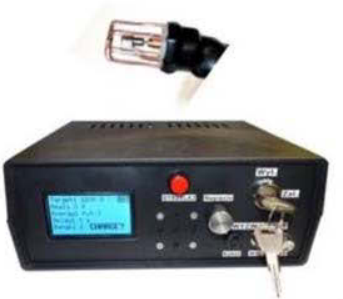

b)

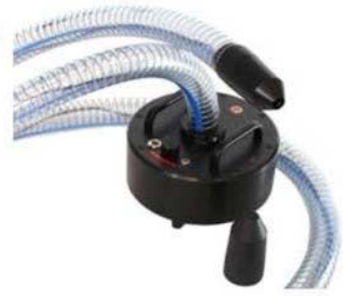

c)
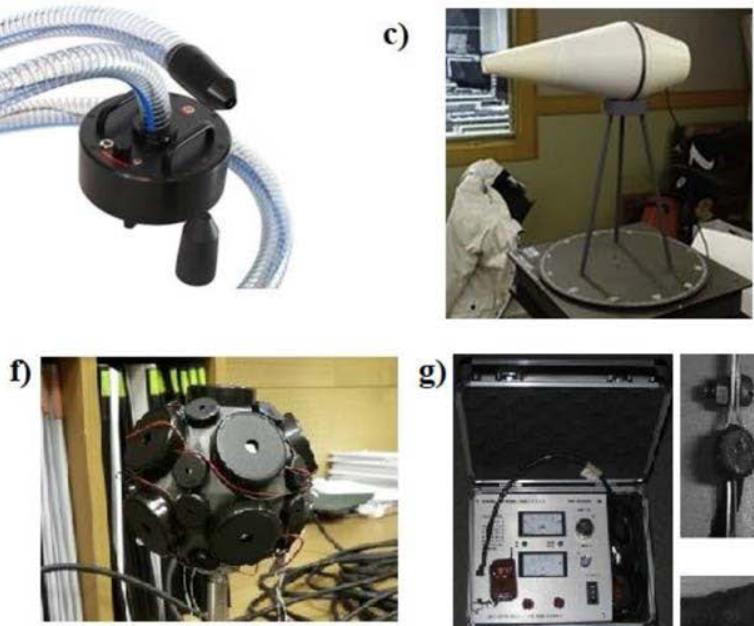

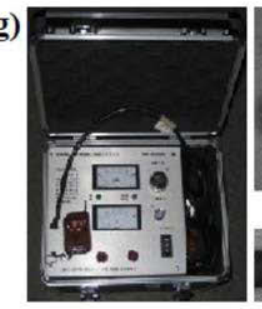

d)

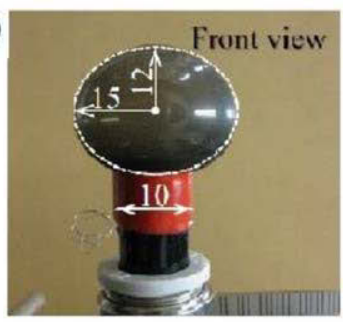

h)

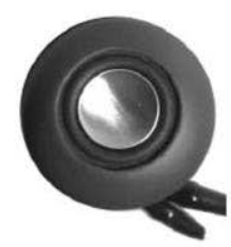

Fig. 2. Examples of omnidirectional sound sources used in acoustic measurements: (a) Microflown low frequency VVS (volume velocity source) [17], (b) Microflown mid-high frequency VVS [17], (c) optimized horn source designed by Ibarra et al. [12], (d) balloon elastomer sound source [13], (e) spark gap sound source [16], (f) piezoelectric scale modeling sound source [14], (g) spark gap sound source for near-field HRTF measurements by $\mathrm{Qu}[6]$, and (h) single speaker near-field sound source by $\mathrm{Yu}$ [3]. 
volume (equivalent air volume, suspension, and diaphragm parameters) were neglected as they do not significantly affect speaker directivity.

\section{MINIATURE OMNIDIRECTIONAL SOUND SOURCES THEORY}

Several analytical methods can be used to describe the behavior of a spherical matrix of loudspeakers. They are usually based on dividing a rigid sphere into a set of elementary sound sources with acoustic velocity applied to their surfaces. Examples of the analytical models are as follows:

1. Multipole Source Model by Zotter [19],

2. Spherical Cap Model by Pasqual [22] and Meyer [23], used by Warusfel [20] and Kassakian [24], and

3. Analytical Dodecahedron Model by Quested [21].

These analytical models are based on accurate mathematical understanding of the problem and are fast and efficient in matrix modeling, making them appropriate for applications such as parametric sources algorithm calibration [20]. However they are not completely appropriate for the design of a physical sound source because the design of electroacoustic devices requires consideration of numerous other factors, such as real dimensions of physical features (e.g., speaker frames and magnets), back-side diaphragm propagation, and electroacoustic parameters of the transducers. Also, the geometry of physical components is usually much more complicated than assumed in theoretical studies.

Another main disadvantage for using the abovementioned analytical methods for near-field calculations is their use of the acoustic far-field zone theorem (Fraunhofer zone simplification [25]). There are several references describing the problems with the formulation of analytical equations in the near-field $[26,27]$ but the boundary between near and far-field is vague [28]. Using far-field-based equations in a near-field can in turn lead to significant errors. Numerical solutions applied in COMSOL Multiphysics software, which was used in this research, are based on HelmholtzKirchhoff integral theorem [29, 30], which is valid in both near and far-field regions [31]. For these reasons FEM and physical modeling were used as the main design tools for an optimized sound source presented in this paper so as to avoid limitations of analytical models.

\section{SIMULATION AND MEASUREMENTS METHODS}

Both laboratory measurements and FEM modeling in COMSOL Multiphysics 5.5 [31] were used in this research. The FEM-simulated 3D radiation patterns were compared with the analytical model by Quested et al. [21] and graphically compared with radiation patterns measured by Leishman [18] to initially validate the model. The comparison between references and our FEM models provided general agreement and correctly validated the simulation models. For the laboratory measurements we used the stand in the form of a microphone arc placed in the anechoic chamber, shown in Fig. 3.

In FEM simulations the observation points were distributed on a sphere of $0.50-\mathrm{m}$ radius around the simulated object, with an angular resolution of $2^{\circ}$ for both azimuth and elevation angles. To model vibrating membranes of the loudspeakers we used a velocity boundary condition applied to selected transducers regions. For experimental verification a microphone array placed on a rotating table in an anechoic chamber was used to simulate the environment from the numerical calculations. The elevation resolution of the microphone array was $15^{\circ}$ within the range of $-60^{\circ}$ to $90^{\circ}$ from the azimuth cut plane of the sound source and the azimuth angular resolution was $5^{\circ}$. The anechoic chamber had a cut-off frequency of around $130 \mathrm{~Hz}$ and the microphone array was constructed with GRAS 46 BE 1/4" microphones connected to Audio Precision APx586 interface.

The most common method for evaluating sound source directivity is the one described in the ISO 354 standard [32]; however it is usually used for diffuse field measurements rather than free-field. There are few methods to assess omnidirectionality of the source for free-field, mostly based on basic statistical functions [33]. So called standard deviation of area-weighted levels (STD AWL $-\sigma_{\mathrm{AWL}}$ ) is adopted in the presented work, following the criteria described in [18] and Eq. (2):

$$
\sqrt{\frac{\sum_{A W L}^{M-1}(f)=0}{\sum_{n=0}^{N-1} S_{m, n}\left[L_{m, n}(f)-\left\langle L_{m, n}(f)\right\rangle_{S}\right]^{2}}},
$$

where $M, N$ are the total number of measurements in 2D space (can be elevation and azimuthal angle), $L_{m, n}(f)$ is SPL at the $m, n$ point, $\left\langle L_{m, n}(f)\right\rangle_{S}$ is the average SPL measured or calculated on the sphere of a given radius, and $S_{m, n}$ is the area of the sphere part corresponding to the point number $m, n$. As such $\sigma_{\mathrm{AWL}}$ is a frequency-dependent objective metric that indicates the uniformity of source strength over all directions.

A large value of $\sigma_{\mathrm{AWL}}$ indicates that the sound source is not omnidirectional for the given frequency band. Leishman et al. [18] suggested use of the $\sigma_{\mathrm{AWL}}(f)$ value of $1 \mathrm{~dB}$ as the threshold of omnidirectionality and the frequency at which this threshold is reached is described as the cut-off frequency of the source for the remainder of this paper. For the wide-band (up to $16 \mathrm{kHz}$ ) measurements it seems to be impossible to aim for matching this condition for the full desired frequency range and use cut-off frequency as a qualification parameter for the source; however we still see $\sigma_{\mathrm{AWL}}(f)$ potential as a parameter to compare the omnidirectional character of the source during the initial design and final evaluation of the source as the strictest omnidirectionality measure available in state-of-the-art.

Normalization by the area covered by the measurement points should compensate for the effect of different numbers of points selected for the calculation while comparing the results between different measurements. This type of statistical method has been widely used for rating the de- 


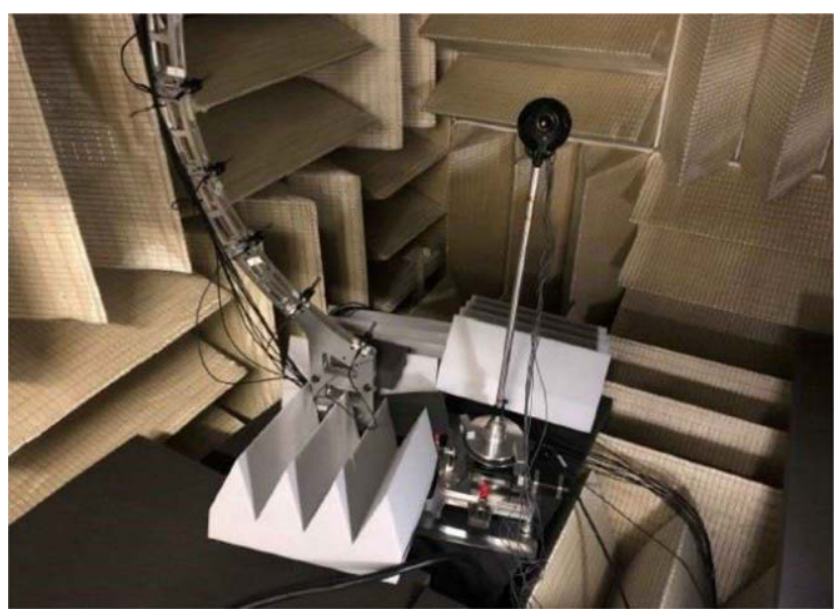

Fig. 3. Measurement setup used for the directivity rating.

viation from the assumed directional pattern [14, 33, 34]. The method presented in [18] is effective as it includes the correction for the area of the measurement "point." This measure should provide the most accurate information on the sound radiation and directivity patterns regardless of the number of receivers used for the directivity measurements or simulations. In each measurement or simulation $\sigma_{\mathrm{AWL}}$ was calculated for narrow frequency bands for points evenly distributed on the sphere around the object. The average value of the $\sigma_{\mathrm{AWL}}$ parameter was also used, calculated from all $N_{f}$ frequency bands used in a given case, in order to provide a single value rating for each configuration, following Eq. (3):

$$
\mu_{S T D}=\frac{\sum_{i=1}^{N_{f}} \sigma_{A W L}(f)}{N_{f}}
$$

\section{DESIGN APPROACH-ENCLOSURE TYPE, SIZE, AND NUMBER OF TRANSDUCERS}

This chapter describes the research on the enclosure size and shape and the number of transducers required to maximize the source omnidirectionality. Different approaches were studied in simulations and experiments to achieve final design requirements and knowledge on optimal solutions for the construction of this type of sound source. We considered the combination of spherical transducers array to achieve close to omnidirectional speaker performance [35, 36] together with a bigger woofer to provide higher SPL in the low-frequency range [37].

\subsection{Number of Transducers and High-Frequency Source Simulations}

The work on this topic as described in [18] only involved polyhedral geometries. Our work specifically considers spherical source configurations as they are most advantageous in minimizing specular reflection and reducing the multi-scattering effect for NF HRTF measurements [8]. The effect of FEM simulations was studied with the $\sigma_{\mathrm{AWL}}$ parameter. Fig. 4 shows set directivity plots for an exam- ple spherical source configuration that were generated via FEM simulations.

The $3 \mathrm{D}$ radiation pattern of this source at $2,000 \mathrm{~Hz}$ shows deviations in spatial response not exceeding $0.7 \mathrm{~dB}$. This increases up to $4 \mathrm{~dB}$ at $4,000 \mathrm{~Hz}$ and to $40 \mathrm{~dB}$ at 8,000 $\mathrm{Hz}$ due to large peaks and notches. These plots illustrate how the radiation pattern of a spherical source composed of multiple elementary sources becomes increasingly complex at higher frequencies, leading to the violation of cut-off frequency in terms of $\sigma_{\mathrm{AWL}}$ as defined earlier.

Our study of the optimal size of the source and the number of transducers was conducted by observing the trend of $\sigma_{\mathrm{AWL}}$ value calculated from a series of FEM simulations with varying design parameters. The simulations were divided into three datasets:

1. constant size of the enclosure- -50 -mm radius, constant size of the transducers- $11 \mathrm{~mm}$, different numbers of transducers-12, 20, and 28;

2. constant size of the enclosure- -50 -mm radius, different sizes of the transducers- $11 \mathrm{~mm}, 14 \mathrm{~mm}$, and $19 \mathrm{~mm}$, constant number of transducers-12; and

3. constant size of the enclosure- 30 -mm radius, different sizes of the transducers- $11 \mathrm{~mm}, 14 \mathrm{~mm}$, and $19 \mathrm{~mm}$; constant number of transducers- 12 .

The source design used for these three sets of simulations is illustrated in Fig. 5. The transducers and enclosure sizes were selected by the transducers' market review and possible usable source size. In the ideal case we should test any combination of transducer number, size, and enclosure size. However this research was firmly aimed to achieve practical design guidelines to construct a real device. For this reason the focus of the investigation remained on general trends related to changing transducer size and number.

Detailed plots of $\sigma_{\mathrm{AWL}}$ over frequency for these simulated configurations are shown in Fig. 6. A lower value of $\sigma_{\mathrm{AWL}}$ at a given frequency correlates to a better omnidirectionality, whereas a lower value of $\mathrm{m}_{\mathrm{STD}}$ indicates a better aggregate performance for the source across all frequencies. A general trend of improving omnidirectionality is observed for 
$\mathbf{N}=12$

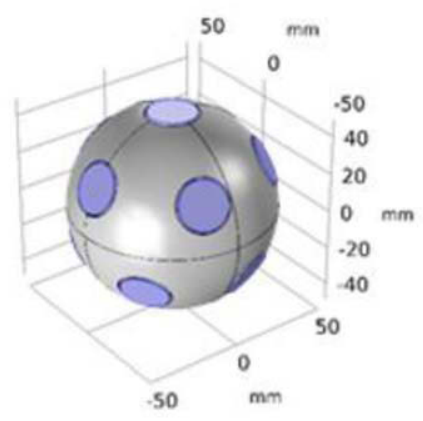

$4000 \mathrm{~Hz}$
$2000 \mathrm{~Hz}$



$8000 \mathrm{~Hz}$
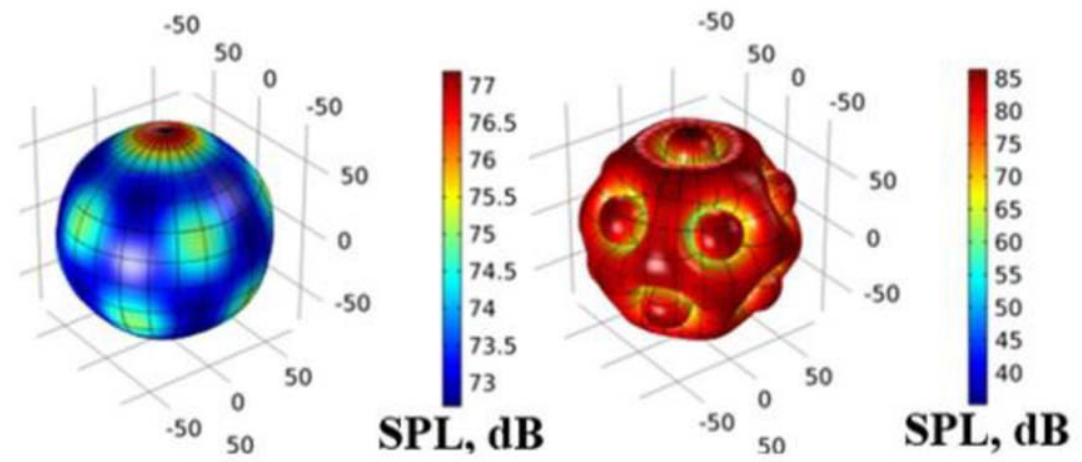

Fig. 4. Selected 3D radiation patterns of 12 transducers spherical loudspeaker matrix with the radius of $50 \mathrm{~mm}$, described in terms of sound pressure level.

increasing the percentage of spherical surface covered by transducer elements. Another simple metric to evaluate the source performance is the cut-off frequency, which can be defined as the lowest frequency at which the source directivity notably deviates from the desired spherical pattern [18]. Above this frequency large increases in $\sigma_{\mathrm{AWL}}$ are observed, which confirms the results from the literature [18, 38]. Leishman defined this as a cut-off frequency, which is the highest frequency at which $\sigma_{\mathrm{AWL}}$ remains below the $1 \mathrm{~dB}$ threshold. The cut-off frequency of $1-1.5 \mathrm{kHz}$ was reported for sound sources over $150 \mathrm{~mm}$ in radius $[39,40]$. Fig. 6(a) indicates that the cut-off frequency systematically increases with the increased number of transducers on the source surface. However, relying only on the cut-off frequency obfuscates the assessment of omnidirectionality in Fig 6(b) and 6(c), where $\sigma_{\mathrm{AWL}}$ dips below $1 \mathrm{~dB}$ even at high frequencies. For these comparisons, observing the trend in $\mu_{\text {STD }}$ is a more reliable evaluation metric, where an increased transducer size leads to a lower $\mu_{\mathrm{STD}}$, indicating improvement in omnidirectionality. It leads to a conclusion that despite cut-off frequency we should always investigate the $\sigma_{\mathrm{AWL}}$ over the frequency range and $\mathrm{m}_{\mathrm{STD}}$ values.

The simulation results also indicate that increasing the number of transducers provides only a small benefit in terms of frequency-averaged omnidirectional performance compared to the other two design variables considered in this study. Increasing the number of transducers may also lead to a larger challenge of finding accurately matching transducer components, which is necessary when accounting for the variation in any factory manufactured components $[14,33]$. A general performance improvement is observed with increasing the size of the transducers; however it also exhibits a tradeoff of stronger peaks across the frequency range of interest, which may be caused by the acoustic interaction between the transducers.

Considering all nine configurations, 19-mm transducers on a 30-mm radius sphere have the highest percentage of spherical surface covered by transducer elements and yielded the lowest $\mathrm{m}_{\mathrm{STD}}$ of $1.9 \mathrm{~dB}$. The trends observed in this parametric simulation study demonstrate that increasing the percentage of source surface covered with transducer elements should be the target principle for the omnidirectional sound source design, which we aim to achieve in this study. By investigating relations between changing elementary source sizes, numbers, and source sizes with FEM, several guiding principles for a near-field source construction were derived. Formulating the priorities in the improvement of source performance under omnidirectionality conditions, we should keep the decreasing source size and increasing transducer size above the transducers' number increments. The next step was to investigate those principles with real elements used for prototyping. 
12 transducers



\section{$11 \mathrm{~mm}$ transducers}

\section{Dataset 2 \\ Sphere $50 \mathrm{~mm}$}

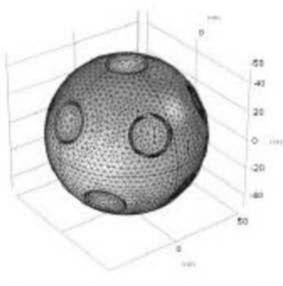

$11 \mathrm{~mm}$ transducers

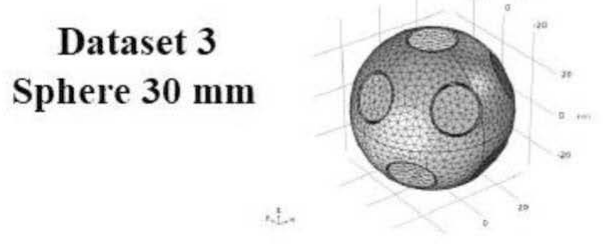

20 transducers

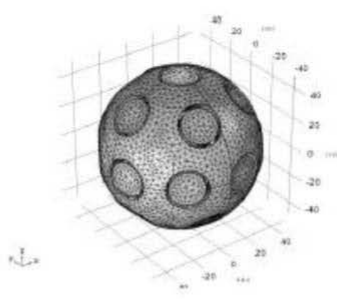

$14 \mathrm{~mm}$ transducers

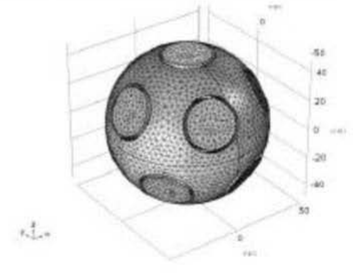

$14 \mathrm{~mm}$ transducers

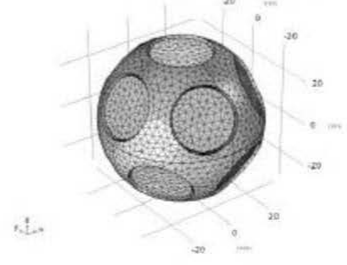

28 transducers



$19 \mathrm{~mm}$ transducers



$19 \mathrm{~mm}$ transducers

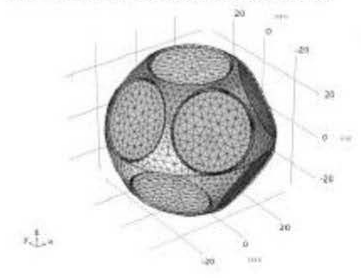

Fig. 5. Simplified spherical source geometries used in initial FEM simulations.

\subsection{Limited Frequency Range of Micro-Speakers-Low-Frequency Extension}

Generally speaking, reducing the overall size of a sound source and extending its acoustic output at low frequencies are diametrically opposite design goals. This is simply described by Eq. (4) [41]:

$$
|p(r)|=\frac{Q \rho c k}{4 \pi r},
$$

which describes the amplitude of sound pressure radiated from a monopole, where the source output is linearly dependent on its strength $Q, \rho$ is medium density, $c$ is speed of sound, and $k$ is a wave number. For a given distance $r$, generating the same pressure level requires a greater source strength at low frequencies than for high frequencies as the $Q$ parameter strongly depends on the effective radiation surface, so the source size. This is a fundamental challenge in increasing the usable frequency range of a small spherical loudspeaker array composed of multiple elementary transducers mounted on the outer surface of a spherical shell. Small elementary transducers are not typically designed to generate a low frequency output without strong distortion. Therefore an alternative method needs to be devised to overcome the challenge associated with this conventional spherical loudspeaker array design.

In a conventional design the size of individual transducers placed on the outer surface determines the overall size of the sound source. The sphere acts as nothing more than a rigid frame on which the transducers are mounted. The newly proposed design exploits the inner volume of the enclosure to place a larger transducer with the ability to effectively drive the acoustic output at lower frequencies while the smaller transducers placed on the outside of the enclosure are used for mid and high frequency reproduction. The overall size of the sound source is determined by the transducers on the outside as described in the previous section. The design assumes that the acoustic output of the inner transducer can be channeled to multiple sound ports, evenly distributed throughout the outer surface of the sphere, to become collated in-phase and form a spherical radiation pattern of a simple source.

The inner assembly of this new design consists of two dynamic loudspeakers and a 3D-printed cylindrical enclosure, which form a sealed volume as the speaker diaphragms are glued to the enclosure while facing each other. In this configuration the sealed volume between the loudspeakers acts as their back-volume while the magnet structures on the 
a)



b)



c)

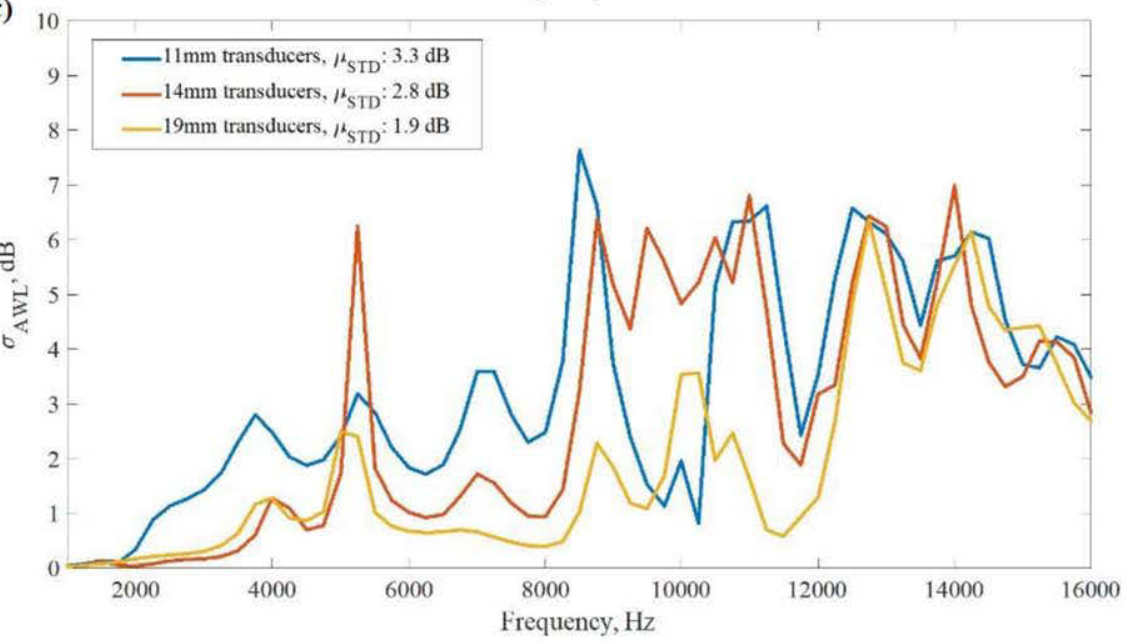

Fig. 6. $\sigma_{\mathrm{AWL}}$ calculated for various configurations of spherical sound sources: (a) varying number of transducers for 50-mm source radius, (b) varying transducer size for 50-mm source radius, and (c) varying transducer size for 30-mm source radius.

backs of the transducers act as rigid diaphragms radiating acoustic pressure. With the goal of tuning the speaker rolloff to the lowest possible frequency, the back-volume was filled with nylon fiber Acousta-Stuf, dedicated to be used inside the cabinets. The preliminary tests provided that filling the volume between the loudspeakers with the nylon fiber increased the output SPL by around $5 \mathrm{~dB}$ in the lowest fre- quency range $(80-150 \mathrm{~Hz})$. It was caused by increasing its effective volume and decreasing speaker attenuation forced by the very small volume inside the enclosure.

The efficacy of the design concept was tested with a COMSOL FEM simulation; Fig.7(a) provides a picture of the initial FEM simulations of the design with the example ported enclosure. By this simulation we wanted to ver- 
a)

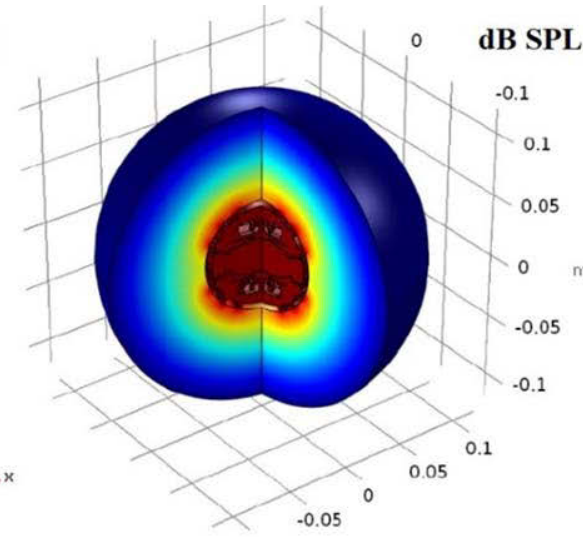

b)



Fig. 7. Initial design of a low-frequency extension device for NF HRTF measurements: (a) simulation and (b) the prototype for directivity testing.

ify the preliminary assumption of a two-drivers subassembly working with small ports. Fig. 7(b) shows the final design with the 3D-printed enclosure and two electrodynamic speakers facing each other in a closed volume. The initial results indicated that the new design can generate an omnidirectional sound field, where the low-frequency roll-off of the inner assembly and the non-uniform distance between the loudspeaker and individual outlet ports limited the frequency bandwidth of omnidirectionality at low and high frequencies, respectively.

The low-frequency extension assembly design was tested with various off-the-shelf transducers and the size of the inner volume to maximize its low-frequency output, with the target of fitting inside a $35 \mathrm{~mm}$-radius spherical enclosure. The optimal design was achieved using the Peerless PMT37N28AL01-04 (hereinafter referred to as P37) dynamic drivers with $37 \mathrm{~mm}$-diameter aluminum diaphragm and a spacing of $15 \mathrm{~mm}$ between the drivers.

Fig. 8 shows the result of directivity and frequency response measurements for this optimum configuration, tested in the anechoic chamber with the method mentioned in SEC. 3 using sine sweep signals. The measurement was taken at $0.5-\mathrm{m}$ distance from the assembly without the surrounding sphere for the high-frequency transducers mount. The maximum allowed power was supplied to the speakers, which was around $4 \mathrm{~W}$ for Peerless P37. The directivity test results showed $\sigma_{\mathrm{AWL}}$ below $1 \mathrm{~dB}$ from $120 \mathrm{~Hz}$ to 2,000 $\mathrm{Hz}$. The omnidirectional performance drop below $120 \mathrm{~Hz}$ is mainly caused by increasing distortions and decreasing SNR below the resonant frequency of the drivers. Considering the anechoic chamber cut-off frequency around 130 $\mathrm{Hz}$, the directivity of the proposed design could be affected by environment eigenmodes.

Following the good electroacoustic practice, the volume of the enclosure should be matched to the number of drivers and their equivalent compliance volume (VAS) parameters; however in this paper the main goal was to keep the source volume as small as possible. For this reason the volume matching was not considered and the size optimization was the main focus. The measured frequency response shows a response roll-off at around $500 \mathrm{~Hz}$, which indicates that two transducers mounted to a small sealed back volume of around $20,000 \mathrm{~mm}^{3}$ do not operate at their maximum efficiency. However the measured response is a notable improvement in terms of the low-frequency output and therefore meets its design goals effectively.

Specifically, the frequency range where the measured SPL at $0.5 \mathrm{~m}$ exceeds 50,55 , and $60 \mathrm{dBSPL}$ is important. The 50-dBSPL threshold can be considered the minimum required SPL for HRTF measurements to provide a usable SNR based on a typical binaural microphone with a noise floor of around $40 \mathrm{dBSPL}$. Higher thresholds of 55 and 60 dBSPL are also considered for scenarios where the source will be used for measurements at larger distances. The requirements defined for transfer function method SNR requirements, described in [42], state that $50 \mathrm{dBSPL}$ should be sufficient for correct HRTF measurements. For the optimized design using P37 drivers the 50-dBSPL threshold was exceeded for frequencies above $96 \mathrm{~Hz}$, which should allow us to reach the $20 \mathrm{~dB}$ SNR required for HRTF measurements [6], and it may be increased by using exponential sweep methods [42, 43].

\subsection{Enclosure Design}

This chapter describes the construction of the spherical enclosure and its effect on the high frequency spatial response of the sound source. In the reviewed references and commercial solutions, datasheets from Brüel \& Kjær [39] and Norsonic [40], there is no information on the construction of the enclosure and separation of the transducers placed outside, on the sphere. State-of-the-art study did not provide an answer to the question if the loudspeakers should be placed in separated chambers or if they can be placed without sealing the volume behind them. In order to answer this question two different geometries were tested (Fig. 9).

For this step of experiments, a new model of sound source enclosure was prepared; mounting elements and venting holes required by the solution explained in SEC. 5.2 were added. The sound ports were placed in between the surfacemounted transducers so they did not block uniform placement of high-frequency transducers. In a sealed version 

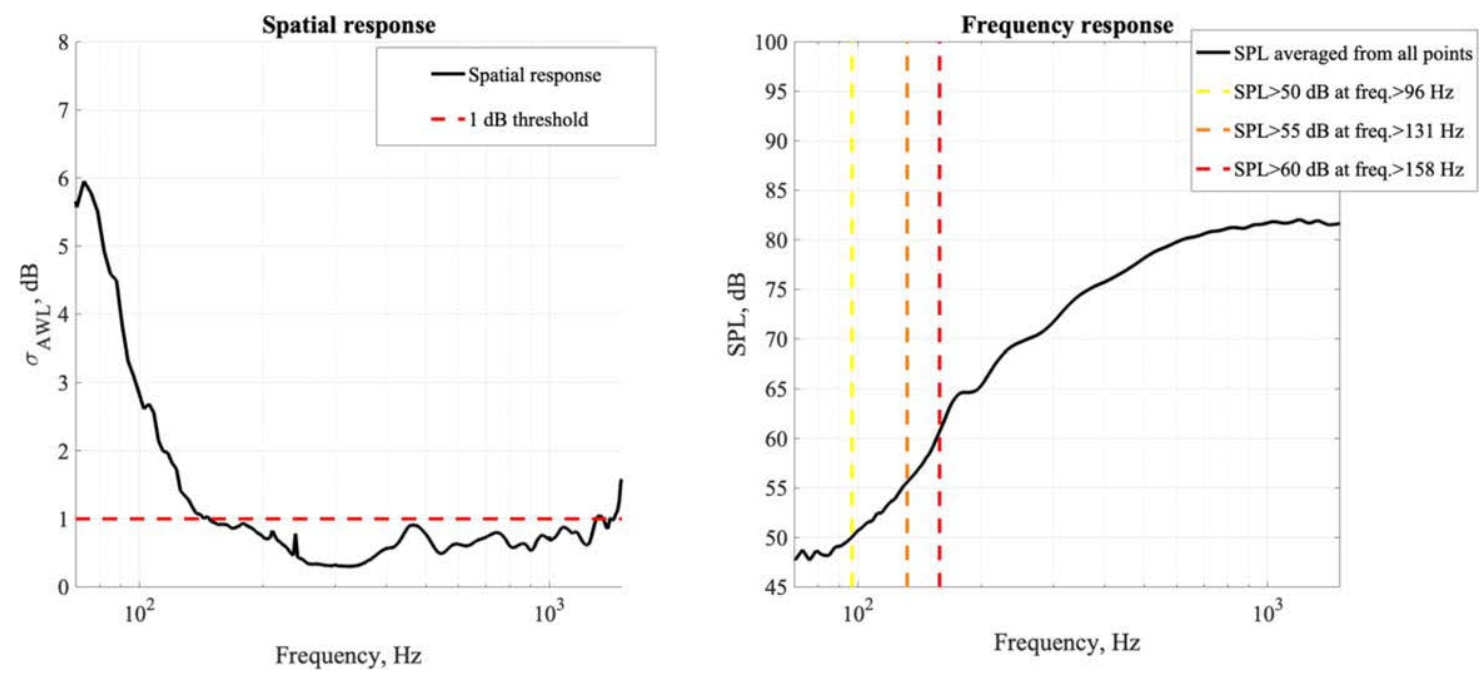

Fig. 8. Directivity and frequency response of the chosen coupled speakers in the $50-16,000 \mathrm{~Hz}$ frequency range.

all high-frequency transducers were sealed in their individual sealed enclosures. Using the non-sealed geometry was considered profitable because it allowed the decrease of the sound source size from $35 \mathrm{~mm}$ in radius (in a sealed version) to $30 \mathrm{~mm}$, which would allow using the source at a smaller distance from the head. However the measurement results shown in Fig. 10 proved that the non-sealed version was much worse, with lower cut-off frequency and overall worse performance in the middle frequency range. This was most probably caused by the interactions between transducers. For this reason, for the final sound source development, the sealed version was selected.

\subsection{Simulation and Measurements Validation}

For the simulation and measurements cross-validation, the direct comparison was made for high-frequency range transducers' final configuration. The results of the directivity assessment for two methods are shown in Fig. 11. As a measurement data set the spatial response results presented in Fig. 10 were adopted. For simulation results an additional model for the sealed geometry type shown in Fig. 9 was created and calculated. Both curves expose the existence of a cut-off frequency parameter, showing that omnidirectional performance starts to degrade around $4 \mathrm{kHz}$. The results differ in absolute value, but considering the measurement features such as dispersion between transducers and measurement uncertainty, the simulation results may be claimed as verified and useful for further investigations in omnidirectional sources design.

\section{TWO-WAY OMNIDIRECTIONAL SOUND SOURCE FOR NEAR-FIELD MEASUREMENTS}

Several complete units of the sound source prototype were constructed following the design methods described previously. Fig. 12 shows photos of the final prototype. Two
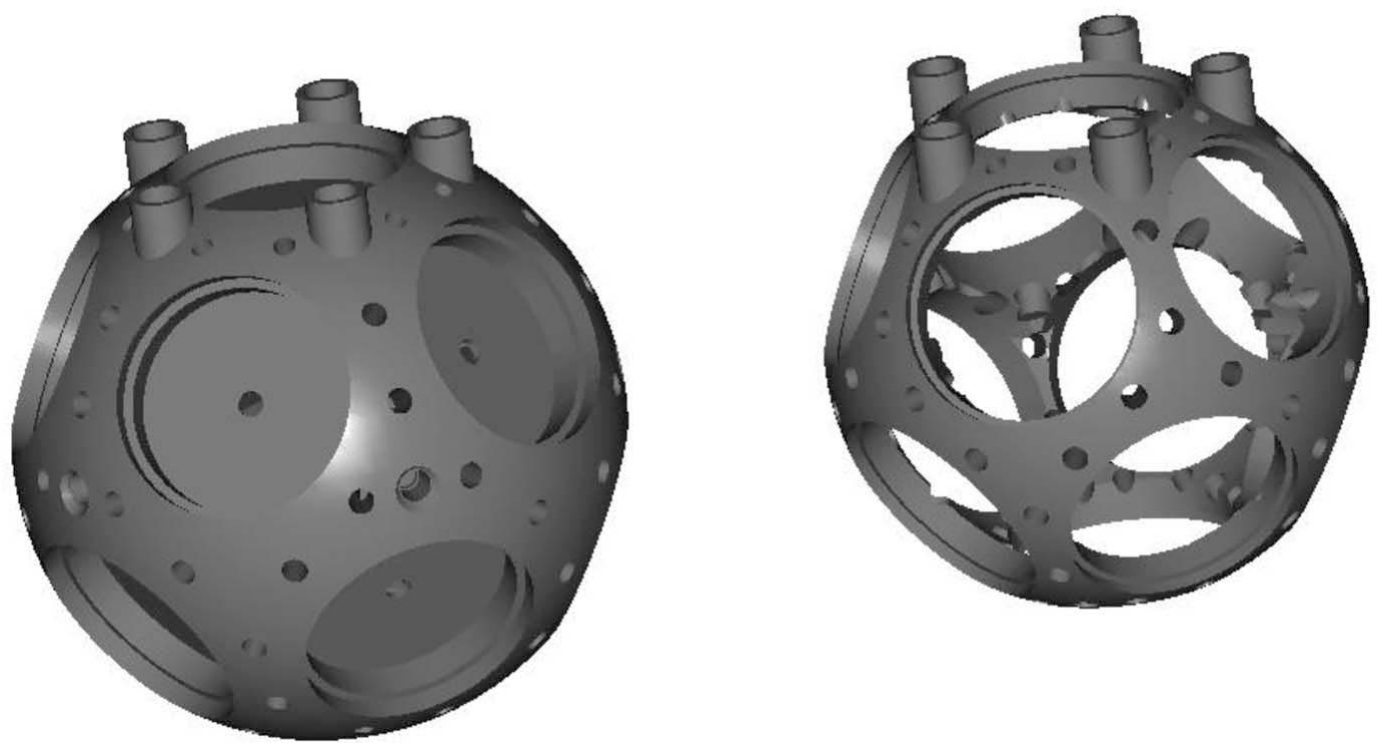

Fig. 9. Sealed (left) and non-sealed (right) geometries of the enclosures used for high-frequency directivity simulations and experiments. 

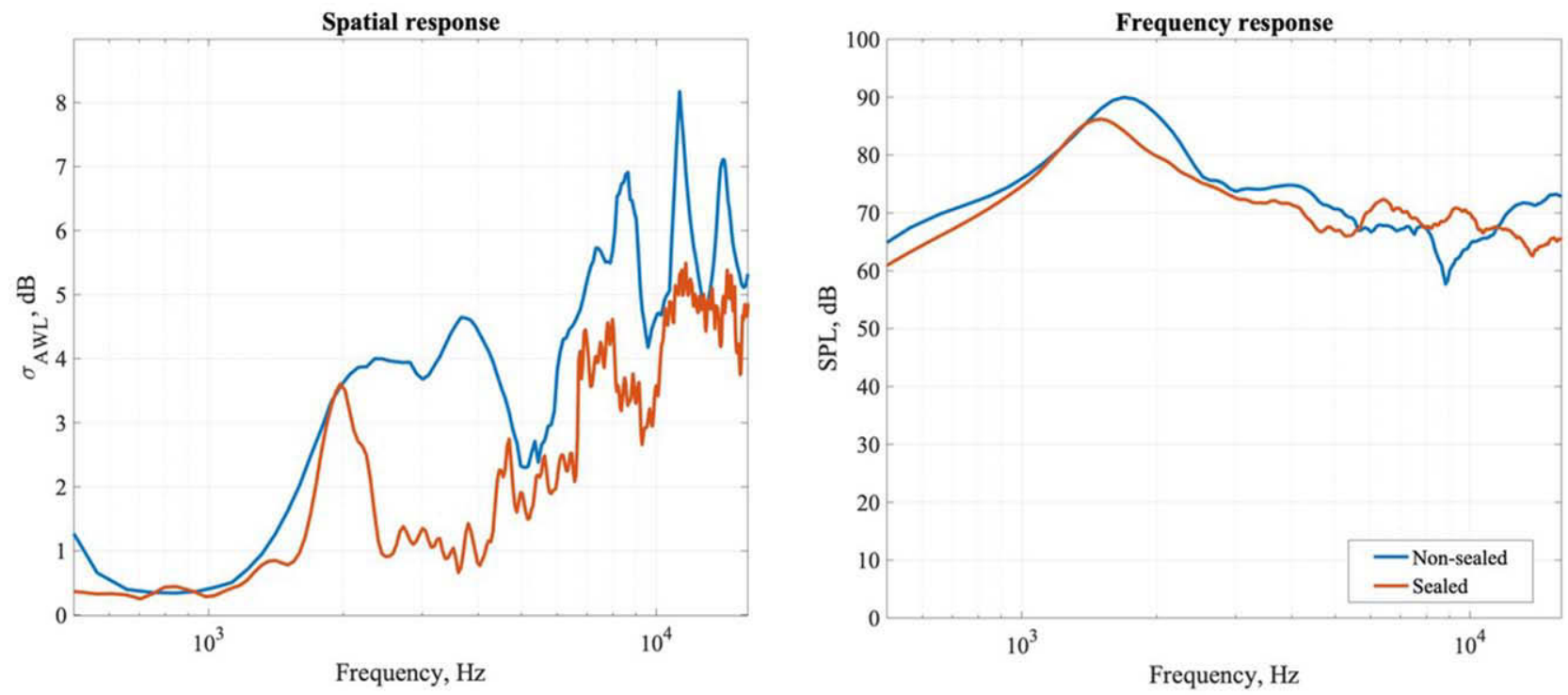

Fig. 10. High-frequency directivity measurements of the sealed and non-sealed versions of the enclosure in the $100-16,000 \mathrm{~Hz}$ frequency range.

transducer types were selected for the final version of the sound source: $28 \mathrm{~mm}$-diameter Visaton K28 on the outside for high frequencies and $37 \mathrm{~mm}$-diameter Peerless P37 on the inside for low frequencies. Apart from their electroacoustic performance the physical dimensions of these two transducer models, including flat membranes, flat magnets, and small frames, made them the most suitable for optimizing the overall size of the full assembly.

The inner device was designed with the biggest possible distance between the diaphragms of low-frequency transducers (P37) to reach the biggest possible internal volume. The inside of the sphere was filled with nylon fiber in order to increase the acoustically effective volume and reduce the reflections inside. The sealed type of the enclosure was used, which is shown in Fig. 12. The final overall size of the enclosure was $34 \mathrm{~mm}$ in diameter. The main limitation for size reduction was the inner chambers to separate the high-frequency transducers. It was necessary to allot enough back-volume for each high-frequency transducer while having enough clearance for the inner assembly to secure enough space for all transducers.

The performance of the full source assembly was validated through directivity and frequency response measurements following the method described in SEC. 3. Each high-frequency transducer was measured separately and summed in post-processing to form a combined response at



Frequency, $\mathrm{Hz}$

Fig. 11. High-frequency directivity simulations vs. measurements comparison for the final design approach in the 1,000-16,000 Hz frequency range. 


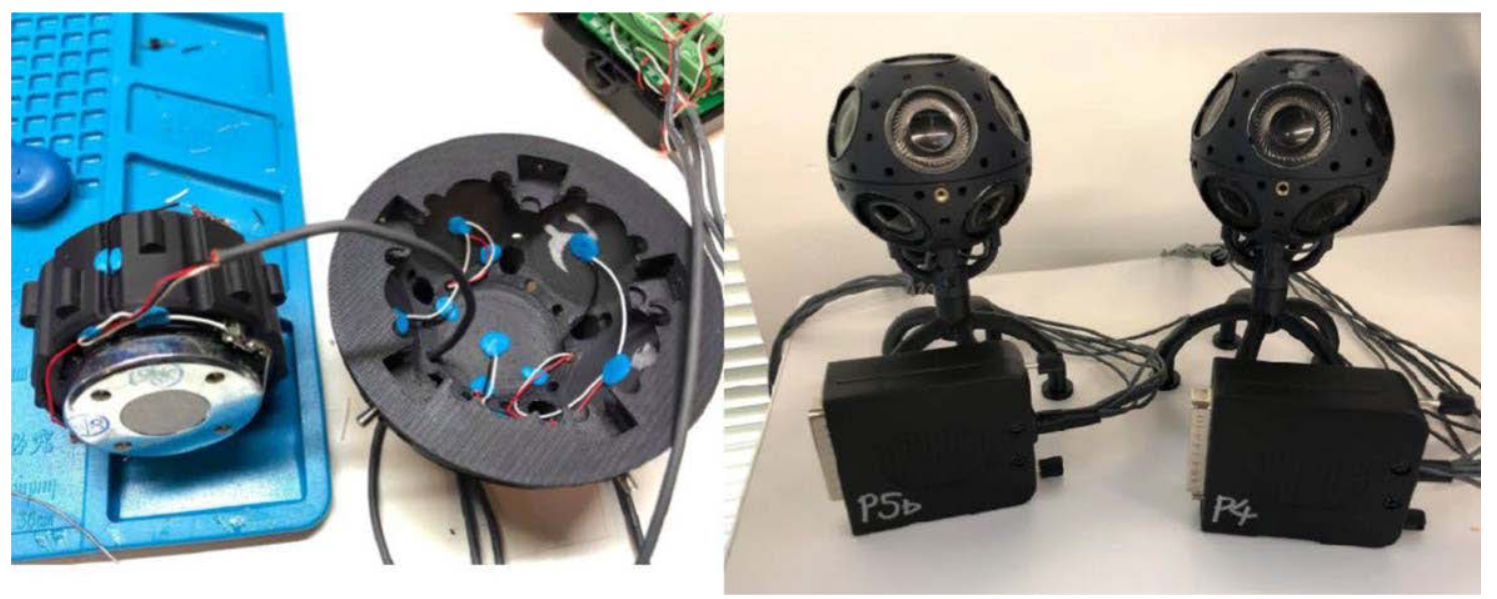

Fig. 12. The inside (left) and full assembly (right) of source prototypes.

each angle. The low-frequency assembly was also measured separately. Fig. 13 displays the average and area-weighted standard deviations of frequency response of low and highfrequency transducers over all measured angles. The results clearly illustrate the functional advantage of each component assembly, where the low-frequency transducers have a notably higher output level and lower standard deviation at frequencies below $1.2 \mathrm{kHz}$, whereas the high-frequency transducers have a higher output level and lower standard deviation at frequencies above $2.5 \mathrm{kHz}$.

To assess the performance of the complete sound source, the results obtained for the two frequency ranges were combined using Linkwitz-Riley filters with the crossover frequency of $3,200 \mathrm{~Hz}$ and $48 \mathrm{~dB}$ slope. This crossover frequency was selected to remove the spike in standard deviation in high-frequency assembly's spatial response at $2,200-2,800 \mathrm{~Hz}$ while maximizing the frequency range where the performance of each source component was superior. Fig. 14 shows the performance of the combined sound source.

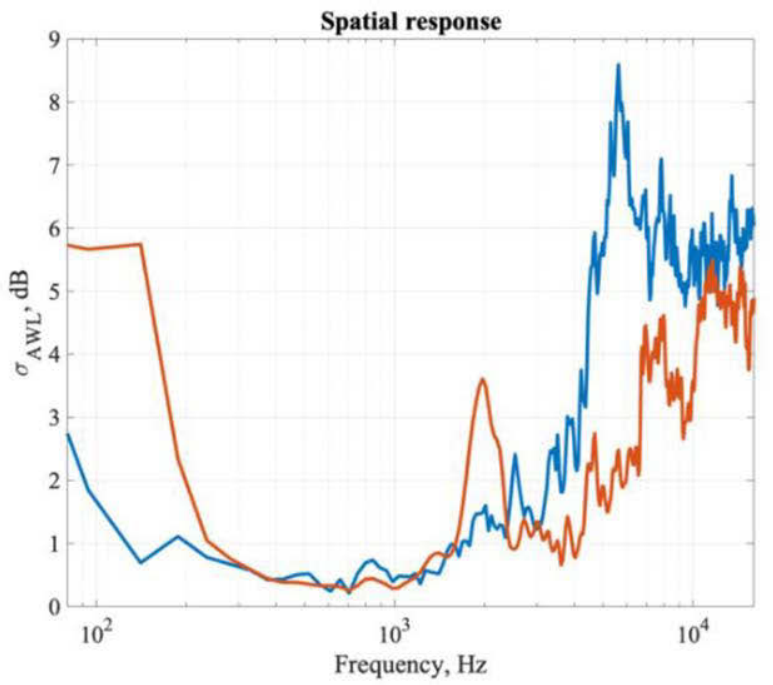

To provide the initial verification of the proposed sound source we conducted the near-field HRTF measurements with the source placed $0.3 \mathrm{~m}$ away from the center of a head. The test object was a 3D printed polyamide artificial head with built-in microphones, which was developed at FRL for internal research. The measured data are postprocessed using FRL's internal processing chain and the resulting HRTF for the left ear are shown in Fig. 15. Only a few measurement results were conducted and are presented here for the initial verification of the proposed sound source, as a full verification is planned in the future to include more artificial objects and human subjects.

These responses demonstrate the constructed source is suitable for the purpose of HRTF measurements. For example the seemingly problematic magnitude response of the combined full-range source at the crossover frequency does not influence the processed HRTF results, as shown in the smooth response at the ipsilateral and frontal directions. Some distortions exist in the higher frequency response for contralateral direction but these are on par with

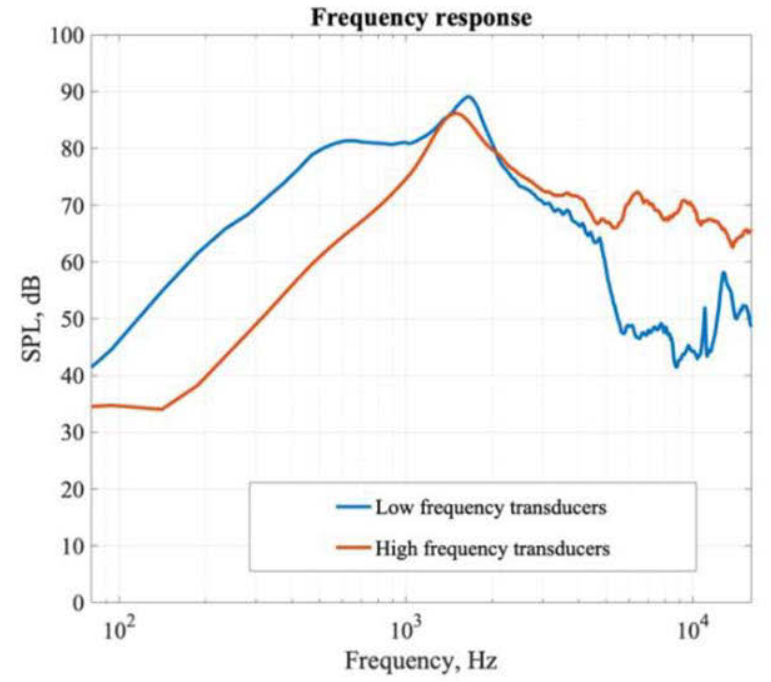

Fig. 13. Frequency and spatial response of high-frequency transducers (on the sphere) and low-frequency transducers (inside the sphere) in the $80-16,000 \mathrm{~Hz}$ frequency range. 

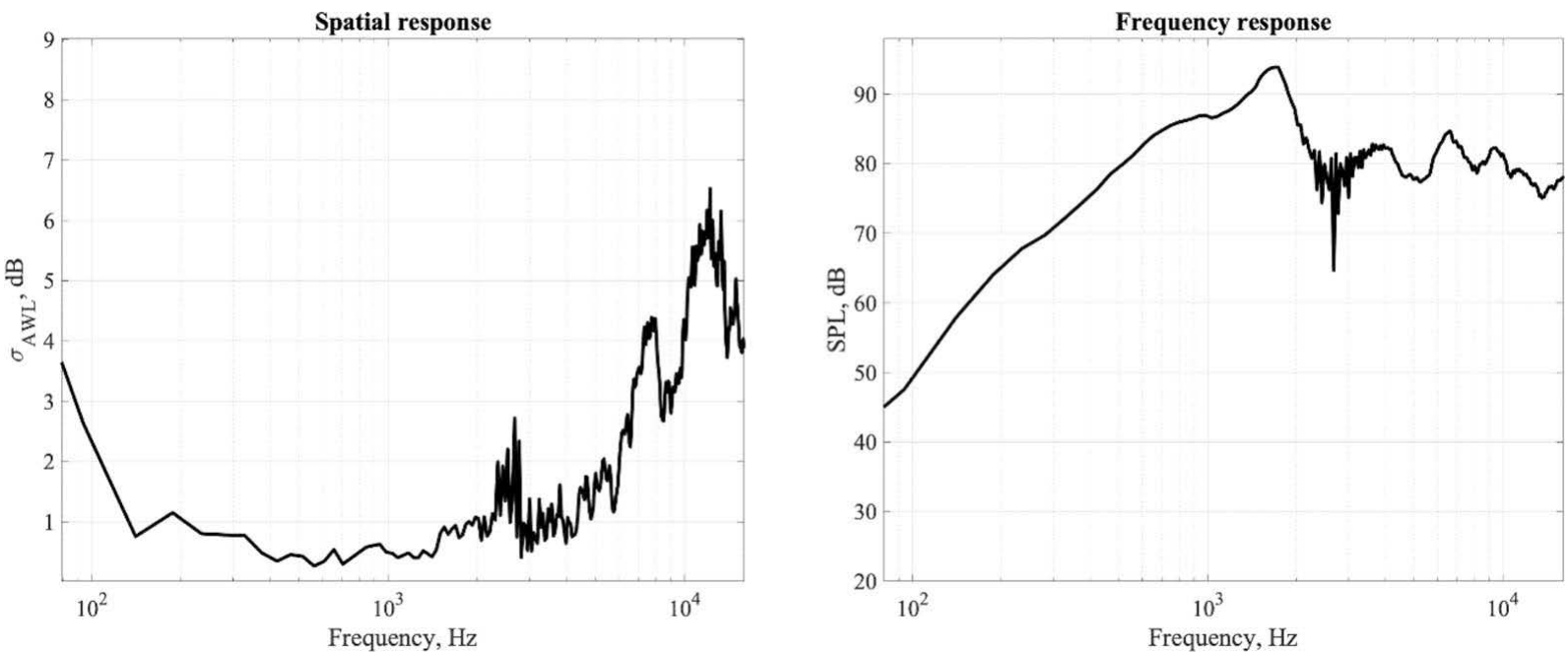

Fig. 14. Frequency and spatial response of the final sound source prototype in the $80-16,000 \mathrm{~Hz}$ frequency range.

or better than results that may be benchmarked in the existing NF HRTF literature [3]. Harmonic distortions from the source are also verified to be effectively removed in the post-processing chain.


\section{DISCUSSION}

Using a target of minimum $50 \mathrm{dBSPL}$ to be useful for near-field HRTF measurement at $0.5 \mathrm{~m}$, the combined

Fig. 15. Example artificial head near-field HRTF measurements, distance $0.3 \mathrm{~m}$, left ear, source facing ipsilateral side (front to the microphone), front side (90 degrees to microphone), and contralateral side (180 degrees to the microphone). 
Table 1. Performance comparison of existing near-field omnidirectional sound sources for HRTF measurements

\begin{tabular}{|c|c|c|c|c|}
\hline Design image & Authors & Mean SPL & $\begin{array}{l}\text { Frequency response } \\
\pm 10 \mathrm{~dB}\end{array}$ & $\begin{array}{l}\text { Declared usable } \\
\text { frequency range }\end{array}$ \\
\hline & $\begin{array}{l}\text { FRL design, } \\
\text { Chojnacki et al. } \\
\text { (2020) }\end{array}$ & $\begin{array}{l}75 \mathrm{~dB} \text { (distance } 0.5 \\
\mathrm{~m})\end{array}$ & $180-16,000 \mathrm{~Hz}$ & $120-16,000 \mathrm{~Hz}$ \\
\hline & $\begin{array}{l}\text { Guang-Zheng et al. } \\
\text { (2008) }\end{array}$ & Not mentioned & $350-20,000 \mathrm{~Hz}$ & $350-20,000 \mathrm{~Hz}$ \\
\hline & $\begin{array}{l}\text { Brungart and } \\
\text { Rabinowitz (1999) }\end{array}$ & $\begin{array}{l}50 \mathrm{~dB} \text { (distance not } \\
\text { mentioned) }\end{array}$ & $200-8,000 \mathrm{~Hz}$ & $200-15,000 \mathrm{~Hz}$ \\
\hline & Qu et al. (2009) & $\begin{array}{l}128 \mathrm{dBA} \text { (distance } \\
0.20 \mathrm{~m} \text { ) }\end{array}$ & $300-24,000 \mathrm{~Hz}$ & $250-24,000 \mathrm{~Hz}$ \\
\hline
\end{tabular}

source meets these criteria in a wide frequency range of $120-16,000 \mathrm{~Hz}$. In comparison to the source presented by Guang-Zheng Yu [8], the lower frequency limit is significantly improved from $350 \mathrm{~Hz}$ to $120 \mathrm{~Hz}$. However it is difficult to define minimum SPL provided by Yu's source in declared frequency range as it is not stated in the reference [4]; the authors only provide the information on the frequency range of $350-20,000 \mathrm{~Hz} \pm 10 \mathrm{~dB}$ without strict reference to lower frequency range. The spatial response of the proposed sound source seems to be very good; however it is hard to be compared to other sound sources as this measure was not used in the previous studies. It should be investigated in further studies and general measurement validation. Some direct comparisons with the other sources with a similar design were placed in Table 1. For comparison needs we analyzed in detail existing data for previous sources' performances, usually presented as plots with limited description. We extracted the information from the plots to define frequency ranges where the magnitude response of a given source does not differ more than $10 \mathrm{~dB}$ from the mean range, but also some declarations from the papers were used where the authors were describing the given frequency range as usable with this kind of the source. The mean SPL parameter was calculated as the arithmetic mean from the data presented on the frequency range plot.

An important explanation for the FRL design data presented in Table 1 is that for two-way source design we can modify the level for two ranges separately, for example lowering the gain for high frequencies, which will then offer decrease of the magnitude at high frequencies and lowering the mean SPL level, so the criteria $\pm 10 \mathrm{~dB}$ will look even better for the source designed in FRL.

The results obtained through this study show improved cut-off frequency of $6 \mathrm{kHz}$ in comparison to $2 \mathrm{kHz}$ obtained in commercial solutions tested in Leishman's work [18]. Direct directivity comparison to most other sources used for near-field HRTF measurements is difficult because they do not report the performance with the same metric. Measurements by $\mathrm{Yu}$ [3] are the primary source of the existing near-field HRTF data on human subjects. These measurements were conducted using a single-speaker sound source with the frequency range of 300-20,000 Hz. The source response dropped by $20 \mathrm{~dB}$ at $200 \mathrm{~Hz}$ compared to the average, leading to an insufficient SNR at the low frequency.

The authors proposed an equalization method to overcome this problem [7]. The spark source measurements by $\mathrm{Qu}$ [6] are another source of near-field HRTF data. In this paper, the authors report reaching over $30 \mathrm{~dB}$ SNR in the $125-\mathrm{Hz}$ octave band. However, the total SPL of the source was measured as $128 \mathrm{dBA}$ at $0.2 \mathrm{~m}$, which makes the source appropriate only for nonhuman subjects like KEMAR. In comparison to these two studies, the two-way source presented in this paper offers a wider usable frequency range down to $120 \mathrm{~Hz}$ with a reasonable SNR without needing an additional equalization scheme. As discussed in SEC. 3, 
the best omnidirectionality, as characterized by the lowest values of $\sigma_{\mathrm{AWL}}$, was achieved below the cut-off frequency of $6 \mathrm{kHz}$. The final operating frequency of $120-16,000 \mathrm{~Hz}$ was determined with respect to the minimum SPL desired for the measurements, which was preliminarily verified in an initial set of near-field HRTF measurements presented in SEC. 5.

It is important to mention that the performance of the proposed source is based on the highest possible SPL output in the desired frequency range, driving each loudspeaker near its input power limit. This may lead to distortion and increased THD for measurements with longer durations. This is a less-critical concern for logarithmic sweep measurements as conducted for our example HRTF measurements, in which the postprocessing chain was designed specifically to remove the influence of harmonics during the calculation of HRIRs [44]. However, this topic of long-term source distortion may be investigated in detail through another study.

\section{CONCLUSIONS}

The paper describes the full design process of designing a sound source to be used in near-field HRTF measurements, based on FEM modeling and physical prototyping. A new design was developed-a sound source that provides a superior frequency range (120-16,000 Hz), usable for near-field HRTF measurements at distances as close as $0.15 \mathrm{~m}$ for a head radius of $0.0875 \mathrm{~m}$ or distances as close as $0.3 \mathrm{~m}$ for a larger head radius of $0.135 \mathrm{~m}$. The efficacy of the proposed method was assessed in a controlled manner with statistical parameters. The questions stated in the introduction were answered-the usability of an inner subassembly for low-frequency range extension was proven. The investigation on the number of transducers and their placement demonstrated an optimal, practical design strategy for the sound source. A simple parameter of $\sigma_{\text {AWL }}$ was used to rate the omnidirectionality of the source and proved to be a good quality metric. The present work offers a solution for acquiring more accurate near-field HRTFs and thus contributes to advancing the spatial audio rendering techniques for AR and VR applications.

\section{REFERENCES}

[1] J. Blauert, Spatial Hearing: The Psychophysics of Human Sound Localization (MIT Press, Cambridge, MA, 1997).

[2] B. S. Xie, X. Zhong, D. Rao, and Z. Liang, "Head-Related Transfer Function Database and Its Analyses," Sci. China G., vol. 50, pp. 267-280 (2007 Jun.). https://doi.org/10.1007/s11433-007-0018-x.

[3] G. Z. Yu, R. Wu, Y. Liu, and B. S. Xie, "NearField Head-Related Transfer-Function Measurement and Database of Human Subjects," J. Acoust. Soc. Am., vol. 143, no. 3, EL194 (2018 Mar.). https://doi.org/10.1121/ 1.5027019 .

[4] G. Z. Yu, B. S. Xie, and D. Rao, "Directivity of Spherical Polyhedron Sound Source Used in NearField HRTF Measurements," Chinese Phys. Lett., vol.
27, no. 12, 124302 (2010 Dec.). https://doi.org/10.1088/ 0256-307X/27/12/124302.

[5] S. Hosoe, T. Nishino, K. Itou, and K. Takeda, "Development of Micro-Dodecahedral Loudspeaker for Measuring Head-Related Transfer Functions in the Proximal Region," in Proceedings of the IEEE International Conference on Acoustics Speech and Signal Processing, pp. 329-332 (Toulouse, France) (2006 May). https:// doi.org/10.1109/ICASSP.2006.1661279.

[6] T. Qu, Z. Xiao, M. Gong, Y. Huang, X. Li, and X. Wu, "Distance-Dependent Head-Related Transfer Functions Measured With High Spatial Resolution Using a Spark Gap," IEEE Trans. Audio Speech Lang. Process., vol. 17, no. 6, pp. 1124-1132 (2009 Aug.). https://doi.org/ 10.1109/TASL.2009.2020532.

[7] D. S. Brungart and W. M. Rabinowitz, "Auditory Localization of Nearby Sources. Head-Related Transfer Functions," J. Acoust. Soc. Am., vol. 106, no. 3, pp. 1465-1479 (1999 Sep.).

[8] G. Z. Yu, B. S. Xie, and D. Rao, "Effect of Sound Source Scattering on Measurement of Near-Field HeadRelated Transfer Functions," Chinese Phys. Lett., vol. 25, no. 8, pp. 2926-2929 (2008 Aug.).

[9] K. M. Bushby, T. Cole, J. N. Matthews, and J. A. Goodship, “Centiles for Adult Head Circumference," Arch. Dis. Child., vol. 67, no. 10, pp. 1286-1287 (1992 Oct.). https://dx.doi.org/10.1136\%2Fadc.67.10.1286.

[10] V. R. Algazi, R. O. Duda, R. Duraiswami, N.A. Gumerov, and Z. Tang, "Approximating the Head-Related Transfer Function Using Simple Geometric Models of the Head and Torso," J. Acoust. Soc. Am., vol. 112, no. 5, pp. 2053-2064 (2002 Nov.).

[11] B. S. Xie, Head-Related Transfer Function and Virtual Auditory Display, 2nd ed. (J. Ross Publishing, Fort Lauderdale, FL, 2013).

[12] D. Ibarra, R. Ledesma, and E. Lopez, "Design and Construction of an Omnidirectional Sound Source With Inverse Filtering Approach for Optimization," HardwareX, vol. 4 (2018 Oct.). https://doi.org/10.1016/j.ohx.2018. $\mathrm{e} 00033$.

[13] N. Hosoya, H. Masuda, and S. Maeda, "Balloon Dielectric Elastomer Actuator Speaker," Appl. Acoust., vol. 148, pp. 238-245 (2019 May). https://doi.org/10.1016/ j.apacoust.2018.12.032.

[14] A. Majchrzak, B. Chojnacki, M. Sobolewska, K. Baruch, and A. Pilch, "The Measurement of Sound Scattering in a 1: 8 Scale-Validation of the Measurement Stand and Procedure," INTER-NOISE NOISE-CON Cong. Conf. Proc., vol. 258, no. 4, pp. 3287-3294 (2018 Dec.).

[15] C. C. J. M. Hak and K. B. A. Bijsterbosch, "Room Acoustic Scale Model Measurements Using a 'Spark Train,", Gerontech. (2009 Jan.).

[16] K. Baruch, A. Majchrzak, B. Przysucha, A. Szelạg, and T. Kamisiński, "The Effect of Changes in Atmospheric Conditions on the Measured Sound Absorption Coefficients of Materials for Scale Model Tests," Appl. Acoust., vol. 141, pp. 250-260 (2018 Dec.).

[17] Microflown, "Sound Sources | VVS" (2020). https:/www.microflown.com/products/sources-vvs/. 
[18] T. W. Leishman, S. Rollins, and H. M. Smith, "An Experimental Evaluation of Regular Polyhedron Loudspeakers as Omnidirectional Sources of Sound," J. Acoust. Soc. Am., vol. 120, no. 3, pp. 1411-1422 (2006 Sep.).

[19] F. Zotter, A. Sontacchi, and R. Höldrich, "Modeling a Spherical Loudspeaker System as Multipole Source," in Proceedings of the 33rd German Annual Conference for Acoustics (DAGA), vol. 33, pp. 221-2 (2007). http://institut17.kug.ac.at/fileadmin/media/iem/altdaten/ projekte/publications/paper/modeling/daga07.pdf.

[20] O. Warusfel, P. Derogis, and R. Causse, "Radiation Synthesis With Digitally Controlled Loudspeakers," presented at the 103rd Convention of the Audio Engineering Society (1997 Sep.), paper 4577. http://www.aes.org/e-lib/browse.cfm?elib=7202.

[21] C. Quested, A. Moorhouse, B. Piper, and B. Hu, "An Analytical Model for a Dodecahedron Loudspeaker Applied to the Design of Omni-Directional Loudspeaker Arrays," Appl. Acoust., vol. 85, pp. 161-171 (2014 Nov.). http://dx.doi.org/10.1016/j.apacoust.2014.03.023.

[22] A. M. Pasqual, J. R. Arruda, and P. Herzog, "A Comparative Study of Platonic Solid Loudspeakers as Directivity Controlled Sound Sources," in Proceedings of the 2nd International Symposium on Ambisonics and Spherical Acoustics, pp. CD:O11-20 (Paris, France) (2010 May). https://hal.archives-ouvertes.fr/hal-00503304.

[23] P. Meyer and J. D. Meyer, "Multi Acoustic Prediction Program (MAPP): Recent Results," Inst. Acoust. (2000).

[24] R. Avizienis, A. Freed, P. Kassakian, and D. Wessel, "A Compact 120 Independent Element Spherical Loudspeaker Array With Programmable Radiation Patterns," presented at the 120th Convention of the Audio Engineering Society (2006 May), paper 6783.

[25] F. Rohrlich, "The Validity of the Helmholtz Theorem," Am. J. Phys., vol. 72, no. 3, pp. 412-413 (2004 Mar.). https://doi.org/10.1119/1.1637041.

[26] S. Weyna, "Investigations of Acoustics Flow and Noise Generation in Circular Duct by Sound Intensity Measurement," Proc. Forum Acust. (2014).

[27] D. Romblom and B. Cook, "Near-Field Compensation for HRTF Processing," presented at the 125th Convention of the Audio Engineering Society (2008 Oct.), paper 7611. http://www.aes.org/e-lib/browse.cfm?elib=14762.

[28] M. S. Kozień, "Acoustic Nearfield and Farfield for Vibrating Piston in Geometrical and Intensity Formulations," Acta Phys. Pol. A, vol. 121, no. 1-A, pp. 132-135 (2012).

[29] A. Bayliss, M. Gunzburger, and E. Turkel, "Boundary Conditions for the Numerical Solution of Elliptic Equations in Exterior Regions," SIAM J. Appl. Math., vol. 42, no. 2, pp. 430-451 (1982 Apr.).

[30] O. A. Godin, "The Kirchhoff-Helmholtz Integral Theorem and Related Identities for Waves in an Inhomogeneous Moving Fluid," J. Acoust. Soc. Am. vol. 99, no. 4, pp. 2468-2500 (1996 Apr.). https://doi.org/10.1121/1. 415524.
[31] GmbH COMSOL, "COMSOL Multiphysics Acoustic Module Documentation, v. 5.5" (2019).

[32] M. L. S. Vercammen, "Improving the Accuracy of Sound Absorption Measurement According to ISO 354," in Proceedings of the International Symposium on Room Acoustics (Melbourne, Australia) (2010 Aug.).

[33] B. Chojnacki, M. Ziobro, and J. Rubacha, "Piezoelectric Omnidirectional Sound Source for Acoustic Scale Measurements in the Ultrasonic Frequency Range [Piezoelektryczne wszechkierunkowe źródło dźwięku do akustycznych badań w skali w zakresie ultradźwięków]," in A. Pilch (Ed.), Studium badawcze młodych akustyków (Wydawnictwo AGH, Kraków, 2016).

[34] C. C. J. M. Hak, R. H. C. Wenmaekers, J. P. M. Hak, and A. P. D. van Luxemburg, "The Source Directivity of a Dodecahedron Sound Source Determined by Stepwise Rotation," in Proceedings of the Forum Acusticum, pp. 1875-1879 (Aalborg, Denmark) (2011 Jun./Jul.).

[35] V. Tarnow, "Sound Radiation From Loudspeaker System With the Symmetry of the Platonic Solids," Brüel Kjar Tech. Rev., vol. 4 (1974).

[36] F. Zotter and M. Frank, "Compact Spherical Loudspeaker Arrays," in Ambisonics: A Practical 3D Audio Theory for Recording, Studio Production, Sound Reinforcement, and Virtual Reality, Springer Topics in Signal Processing, vol. 19, pp. 153-170 (Springer, Cham, New York, NY, 2019). https://doi.org/10.1007/978-3-030-17207 -7 7.

[37] J. Borwick, Loudspeaker and Headphone Handbook, 3rd ed. (Routledge, Oxfordshire, England, 2001).

[38] A. M. Pasqual, J. R. de França Arruda, and P. Herzog, "Application of Acoustic Radiation Modes in the Directivity Control by a Spherical Loudspeaker Array," Acta Acust. United Acust., vol. 96, no. 1, pp. 32-42 (2010 Jan./Feb.).

[39] Brüel \& Kjær, "OmniPower Sound Source” (2019). https://www.bksv.com/en/products/transducers/acoustic/ sound-sources/omni-power-light-4292.

[40] Norsonic, "Dodecahedron Loudspeaker Nor276" (2019). https://web2.norsonic.com/product_single/ dodecahedron-loudspeaker-nor276/.

[41] D. A. Russell, J. P. Titlow, and Y. -J. Bemmen, "Acoustic Monopoles, Dipoles, and Quadrupoles: An Experiment Revisited," Am. J. Phys., vol. 67, no. 8, pp. 660664 (1999 Aug.).

[42] S. Müller and P. Massarani, "Transfer-Function Measurement With Sweeps," J. Audio Eng. Soc., vol. 49, no. 6, pp. 443-471 (2001 Jun.). http://www.aes.org/e-lib/ browse.cfm?elib $=10189$.

[43] P. Dietrich, B. Masiero, and M. Vorländer, "On the Optimization of the Multiple Exponential Sweep Method," J. Audio Eng. Soc., vol. 61, no. 3, pp. 113-124 (2013 Mar.).

[44] A. Torras-Rosell and F. Jacobsen, "A New Interpretation of Distortion Artifacts in Sweep Measurements," $J$. Audio Eng. Soc., vol. 59, no. 5, pp. 283-289 (2011 May). http://www.aes.org/e-lib/browse.cfm?elib=15929. 


\section{THE AUTHORS}

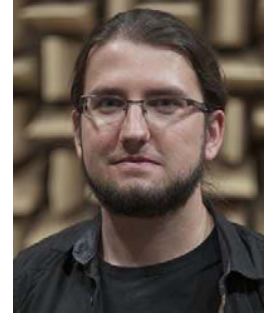

Bartlomiej Chojnacki

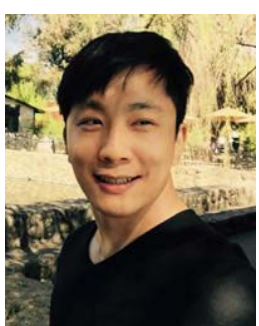

Sang-Ik Terry Cho



Ravish Mehra
Bartlomiej Chojnacki is the Acoustic Research Engineer and Scientist for the AGH University of Science and Technology in Poland, Cracow. In 2018 he was a Research Intern in Facebook Reality Labs, Redmond, WA. He received a master's degree in acoustical engineering at AGH University in 2018, working on architectural and small rooms acoustics. He is now pursuing a $\mathrm{PhD}$ degree on omnidirectional sound sources development. In the industry he is working with the acoustic treatment manufacturers such as Mega-Acoustic or Soundway Acoustics, working as an R\&D engineer and manager. Bartlomiej Chojnacki is an active member of international audio-related organizations, such as the Audio Engineering Society or European Acoustics Association.

Sang-Ik Terry Cho is the Acoustic Engineering Manager for the Research Audio Team at Facebook Reality Labs, leading a team of acoustic research engineers and DSP engineers. He received a bachelor's degree in electrical engineering at The Cooper Union in 2007 and $\mathrm{PhD}$ in acoustics at Pennsylvania State University in 2012 with research on FDTD simulation of low-amplitude sonic boom propagation around building structures. His work currently focuses on the system integration of the latest audio research advancements to AR and VR devices, changing the way speech and audio media are consumed in people's daily lives.

Ravish Mehra is the Research Science Lead for the Audio Team at Facebook Reality Labs responsible for developing novel audio techniques to push the state-of-the-art for audio in VR and AR. He completed his PhD in Computer Science at the University of North Carolina at Chapel Hill in the field of acoustics and spatial audio. In his doctoral work, he worked on novel physically based simulation techniques for simulating complex acoustic phenomena rising out of propagation of sound waves in large environments. His research interests span the fields of audio, acoustics, signal processing, and virtual and augmented reality. Dr. Mehra's work in acoustics and spatial audio has generated considerable interest in the audio community and his sound propagation and spatial sound system has been integrated into virtual reality systems (Oculus HMD) with demonstrated benefits. 